\title{
Generation and characterization of novel stromal specific antibodies
}

\author{
Sapna HALDER ${ }^{1, *}$, Debbie L. HARDIE ${ }^{2, *}$, Dagmar SCHEEL-TOELLNER ${ }^{2}$, Mike SALMON ${ }^{2}$, Christopher D. \\ BUCKLEY ${ }^{2}$ \\ ${ }^{1}$ Prostate Cancer Research Centre, Institute of Urology (3 ${ }^{\text {rd }}$ floor), University College London, 67 Riding House Street, \\ London W1W $7 E J$, UK \\ ${ }^{2}$ Rheumatology Research Group, MRC Centre for Immune Regulation, Institute of Biomedical Research $\left(3^{\text {rd }}\right.$ or $4^{\text {th }}$ floor), \\ University of Birmingham, Birmingham B15 2TT, UK
}

\begin{abstract}
Rheumatoid synovial fibroblasts were used as an immunogen to produce monoclonal antibodies selected for their reactivity with stromal cell antigens. Mice were immunised with low passage whole cell preparations and the subsequent hybridomas were screened by immunohistochemistry on rheumatoid synovium and tonsil sections. The aim was to identify those antibodies that recognised antigens that were restricted to stromal cells and were not expressed on CD45 positive leucocytes. A significant number of antibodies detected antigen that identified endothelial cells. These antibodies were further characterised to determine whether the vessels identified by these antibodies were vascular or lymphatic. From five fusions clones were identified with predominant reactivity with: 1) fibroblasts and endothelial cells; or 2) broad stromal elements (fibroblast, endothelium, epithelium, follicular dendritic cells). A fibroblast-specific antibody that did not also identify vessels was not generated. Examples of each reactivity pattern are discussed.
\end{abstract}

Keywords: stromal, mesenchymal, non-haematopoietic, rheumatoid, fibroblasts, CD45.

\section{INTRODUCTION}

A variety of methods have been used to grow, select and clone antibody-producing hybridomas since the first derivation of monoclonal antibodies (mAbs) of defined specificity in 1975 [1]. There are many markers for leucocyte subsets and specifically those of the lymphocyte lineage and its subdivisions. However there are currently very few specific markers for monitoring and isolating fibroblast populations or for discriminating between fibroblast subsets. One approach to this problem has been gene expression profiling which has conclusively demonstrated that fibroblasts from different sites are differentiated in a site-specific manner [2,3]. We have adopted a more classical approach by generating mAbs specific for stromal cells, including rheumatoid synovial fibroblasts.

\footnotetext{
*Correspondence: Sapna Halder ${ }^{1}$, Debbie Hardie ${ }^{2}$.

${ }^{1}$ E-mail: s.halder@ucl.ac.uk

${ }^{2}$ E-mail: d.1.hardie@bham.ac.uk
}

\section{MATERIALS AND METHODS}

\section{Strategy}

A total of 5 fusions were performed with 3 (BR-26, -27, -28) using fibroblasts taken from frozen stocks derived from a single patient and injected into mice and 2 (BR-32, -33) using fibroblasts taken from culture derived from different patients for each fusion and injected into mice. Each clone obtained was initially screened by immunofluorescence on rheumatoid sections; those with reactivity were then screened on tonsil sections and if there was no broad leucocyte expression the clone was taken forward for expansion and further subsequent rheumatoid and tonsil screening to ensure continued $\mathrm{mAb}$ expression. Fibroblasts are the predominant cell type in the synovial sections and it was not always possible to discard clones that were broadly reactive on this first screen. The secondary screen on sections of tonsil was successful at identifying those unwanted clones that may, for example, have reactivity with MHC Class I or had broad reactivity with haematopoietic cells. To avoid detection of idiotypic antibodies the synovial tissue used for screening was not from the same source as used for immunisations; in addition as fibroblasts from the same tissue show similar gene expression profiles [2, $3]$, it was considered unlikely that it was necessary to screen clones with fibroblasts derived from the same patient as used for immunisation. Flow cytometry was performed on selected mAbs to ensure that the mAbs were not broadly expressed on the surface of 
leucocytes from tonsil and blood. The isotype of the mAbs was determined by ELISA as per instructions (ISO2, Sigma, UK).

\section{In Vitro propagation of Fibroblasts}

Primary human rheumatoid synovial fibroblasts were obtained from patients undergoing knee/elbow replacement surgery. Synovial tissue was manually cut into small pieces with a sterile scalpel under sterile conditions. The tissue was washed in phosphate buffered saline (PBS) (Sigma, UK) twice and collagenase (Sigma, UK) was added at concentration of $0.1 \mathrm{mg} / \mathrm{ml}$ and tissue was agitated for $16-$ $24 \mathrm{~h}$ to ensure tissue digestion. After digestion the tissue was mechanically disaggregated and sieved then tissue placed in Petri dish with cover slip on top of tissue with medium to allow fibroblast growth. Fibroblasts were propagated in RPMI supplemented with $10 \%$ foetal calf serum (FCS), 1\% L-glutamine, 1\% penicillin-streptomycin (Sigma, UK). Cells were passaged 4 times and then either frozen (in 10\% dimethyl sulfoxide DMSO/FCS (Sigma, UK)) or used directly from culture for immunisation. Whilst fibroblasts are a major component of synovial tissue, other cell types were not formally excluded from the preparations. The frozen cells were derived from one patient and the cells used from culture were derived from 2 different patients.

\section{Fusion}

Female 6-week-old Balb/c mice were immunised subcutaneously at weekly intervals for 4 weeks with $1 \times 10^{6}$ low passage (passage 4) whole rheumatoid synovial fibroblasts. The fusion was performed as per instructions with the kit used (Clona Cell-HY kit, Stemcell, Canada). Briefly, splenocytes from the mice were fused with immortal NSO-1 cells (kindly donated by Margaret Goodall, University of Birmingham, UK) with the addition of polyethylene glycol (Clona Cell-HY kit, Stemcell, Canada). The resulting mix was grown in selective agar (ClonaCell-HY kit, Stemcell, Canada) and desired clones expanded after screening by immunofluorescence.

\section{Immunofluorescence}

Rheumatoid synovium and tonsil sections $(5 \mu \mathrm{m})$ were fixed in acetone (Sigma, UK) for $10 \mathrm{~min}$. $50 \mu \mathrm{l}$ of mAb supernatant was added to each section and incubated in a moist chamber for $20 \mathrm{~min}$. The negative control was the media used for hybridoma growth (media E from ClonaCell-HY kit, Stemcell, Canada). The sections were washed in phosphate buffered saline (PBS) (Sigma, UK), $50 \mu 1$ anti-mouse fluorescein isothiocyanate (FITC) (Caltag, USA) added and incubated as previously then washed in a PBS bath for $20 \mathrm{~min}$. The slides were counterstained with propidium iodide solution (1 $\mu \mathrm{g} / \mathrm{ml}$ ) (Sigma, UK) for $1 \mathrm{~min}$, washed then mounted in anti-fade reagent (2.4\% 1,4-Diazabicyclo [2.2.2] octane (DABCO) (Aldrich, Gillingham, England) in glycerol (Fisons Scientific, Loughborough, UK) $\mathrm{pH}$ 8.6. For multiple colour immunofluoresence antibodies BR27 4.11 and BR27 9.1 were used in combination with 8D6 (1:50 CD320 mAb kindly donated to HLDA8 by Dr Li LI, Alton Ochsner Clinic Foundation, LA, USA), von Willebrand factor (vWF) (1:500 A0082 DAKO, UK) and CD45-APC (1:100 340910 Becton-Dickinson, Oxford, UK) and detected with biotin anti-mouse IgM (1:50 102008 Southern Biotechnology, UK) in combination with streptavidinAlexa $^{\text {TM} 488 ~(1: 100 ~ S-32354 ~ I n v i t r o g e n, ~ U K), ~ a n t i-m o u s e ~ I g G 1 ~}$ tetramethyl-rhodamine (1:50 1070-03 Southern Biotechnology, UK) and anti-rabbit 7-amino-4-methylcoumarin-3-acetic acid (1:200 711156-152 Stratech Scientific Ltd, UK) respectively. BR28 30.10 was detected using the biotin/streptavidin system as above, and used in combination with vWF - detected as above and LYVE-1 (mouse IgG1 clone $8 \mathrm{C}$ kindly donated by David Jackson, MRC Immunology Unit, John Radcliffe Hospital, Oxford, UK). Images were captured using a laser-scanning confocal microscope (LSM 510 system Zeiss, Germany).

\section{Surface staining}

Staining procedures were carried out using 96 U bottom multiwell plates (Biomedical Laboratory Supplies, Birmingham, UK). Diluent at all stages was $0.15 \mathrm{M}$ phosphate buffered saline $\mathrm{pH} 7.4$ (PBS) containing 5\% foetal calf serum (FCS) (Northumbrian Biological Industries, UK), 5\% normal goat serum (Gibco-BRL, Paisley, Scotland) and $0.05 \%$ sodium azide (Sigma, UK). $200 \mu$ of pre-washed target samples containing $0.25 \times 10^{6}$ cells were aliquoted into multiwell plates. Cells were spun at $300 \times \mathrm{g}$ for $3 \mathrm{~min}$. Supernatants were aspirated then $25 \mu \mathrm{l}$ of appropriately diluted antibody was added and well contents were mixed. The plates were covered and placed on ice on a shaker for $30 \mathrm{~min}$ then washed twice with diluent. $25 \mu \mathrm{l}$ of appropriately diluted anti-mouse FITC (Caltag, Burlingame, CA, USA) was added to each well and the well contents mixed. The plates were covered and placed on ice on a shaker for $30 \mathrm{~min}$. The plates were then washed as described above. $25 \mu 1$ of $10 \%$ normal mouse serum (The Binding Site, Birmingham, UK) in diluent was added for $15 \mathrm{~min}$ on ice as a blocking stage, the plates were spun and supernatants were removed as above.

Tonsil cells were then stained with anti-human CD19 allophycocyanine (APC) (diluted 1 in 50), CD38 phycoerytherin (PE) (diluted 1 in 50) and CD3 CyChrome ${ }^{\mathrm{TM}}$ (diluted 1 in 25) (BectonDickinson, Oxford, UK); whilst blood cells were stained with CD19, or CD20 plus CD21 (all diluted 1 in 50) APC, CD14 PE (diluted 1 in 50) and $\mathrm{CD} 3 \mathrm{CyChrome}^{\mathrm{TM}}$ (diluted 1 in 25) (Becton-Dickinson, Oxford, UK). $25 \mu$ l of the conjugate mix was incubated with the cells for 30 minutes on ice on a shaker then washed as above. $100 \mu \mathrm{l}$ of diluent was added to each well and the contents of the well were transferred to a $75 \mathrm{~mm}$ FACs tube (Alpha Laboratories, Eastleigh, UK); to each FACs tube $500 \mu 1$ of $1 \%(\mathrm{w} / \mathrm{v})$ paraformaldehyde (BDH, Poole, England) in PBS pH 7.4 was added. Samples were read on a Becton Dickinson Facscalibur (Oxford, UK) fluorescence activated cell analyser (FACs). Cells were selected by gating on forward (size) and side (granularity) scatter. A minimum of 5,000 events within this gate was analysed.

\section{Blood leucocyte preparation}

Leucocyte enriched preparations of buffy coat cells from healthy donors were obtained from the Birmingham Blood Transfusion Service. These preparations had been depleted of platelets and contained sodium citrate as anti-coagulant. The buffy coat was diluted 1 : 1 with PBS containing $20 \mathrm{U}$ per $\mathrm{ml}$ of Heparin (CP Pharmaceuticals, Wrexham, UK) and $0.1 \%$ sodium azide. This was layered onto a Ficoll-Paque density gradient (Pharmacia-Biotech, Uppsala, Sweden), using a cell suspension to density gradient volume ratio of $\sim 3: 2$. The preparation was centrifuged at $450 \mathrm{~g}$ for $25 \mathrm{~min}$ at room temperature and the interface between the density gradient and blood sample that contains the leucocytes was harvested into washing buffer at a ratio of 1 part cell suspension to 3 parts washing buffer (RPMI 1640 Hepes buffered medium containing $2 \%$ (v/v) FCS). Cells were washed, firstly at $400 \times \mathrm{g}$ for $7 \mathrm{~min}$, then a second wash of $300 \times \mathrm{g}$ for $3 \mathrm{~min}$. Samples were checked for platelet contamination and, if required, a 
Tab. 1 Hybridoma screening strategy

\begin{tabular}{ccccc}
\hline Fusion & $\begin{array}{c}\text { Total number of } \\
\text { colonies picked }\end{array}$ & $\begin{array}{c}\text { No of clones selected after initial } \\
\text { rheumatoid screen }\end{array}$ & $\begin{array}{c}\text { No of clones selected after } \\
\text { initial tonsil screen }\end{array}$ & $\begin{array}{c}\text { No of clones selected after } \\
\text { subsequent tonsil screen }\end{array}$ \\
\hline BR-26 & 219 & 31 & 9 & 8 \\
BR-27 & 221 & 22 & 8 & 8 \\
BR-28 & 601 & 52 & 14 & 13 \\
BR-32 & 765 & 17 & 11 & 4 \\
BR-33 & 107 & 5 & 2 & 1 \\
\hline
\end{tabular}
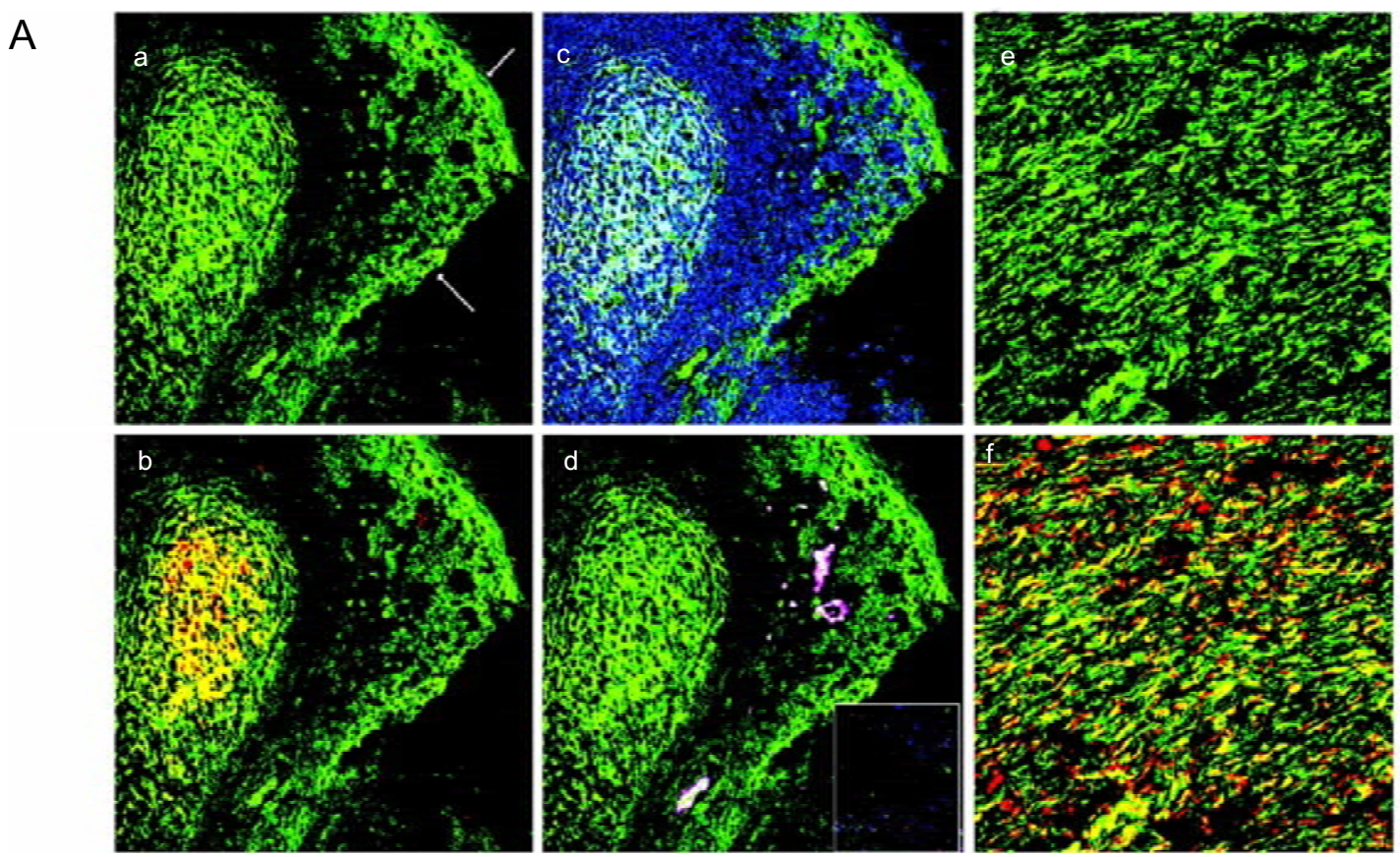

\section{B}

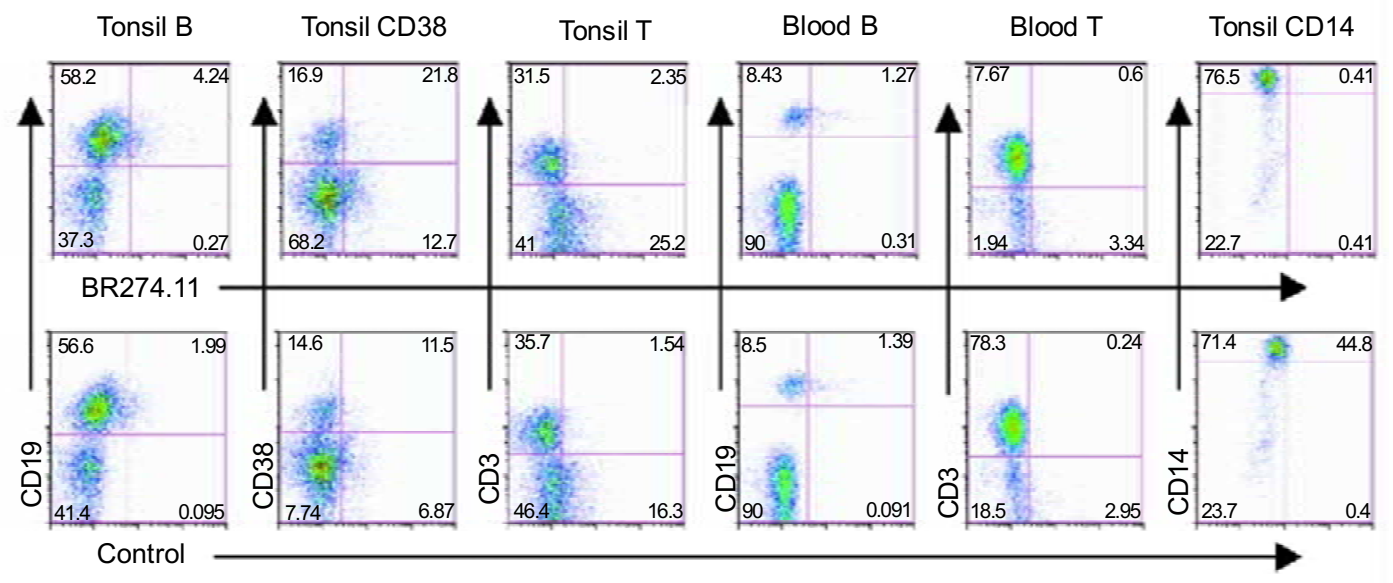

Fig. 1 Broad stromal expression by mAb BR27 4.11. (A) BR27 4.11 (green) staining in tonsil (a-d) and rheumatoid synovium (e,f) in comparison with (b) 8D6 (red) (CD320); (c) CD45; (blue) (d) vWF (pink) and (f) nuclei (red). Co-incident staining appears as yellow (b and f), cyan (c) or white (d). BR27 4.11 has extensive staining of follicular dendritic cells $\left(8 \mathrm{D} 6^{+}\right.$) that are also CD45 $5^{+}$other CD45 cells are not BR27 4.11 $1^{+}$. BR27 4.11 also stains $\mathrm{VWF}^{+}$vessels and epithelium (arrowed). Inset in (d) is negative control of secondary antibodies. Magnification: $\times 17.5$. (B) Lower panels: FACS data showing no surface expression of this antibody on forward and side scatter

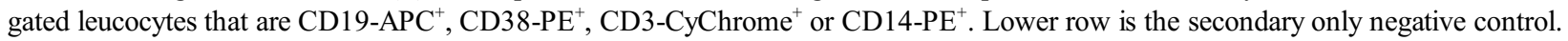


further wash and spin at $300 \times \mathrm{g}$ for 3 min was carried out. These cells were then used for flow cytometric analysis; lymphocytes or monocytes being selected by gating on forward and side scatter parameters.

\section{Tonsil leucocyte preparation}

Normal human palatine tonsils were collected immediately after surgical removal into sterile universal bottles (Appleton Woods, Birmingham, UK). Tonsils were placed into petri dishes (Appleton Woods) containing washing buffer. Excess fibrous tissue was re- moved and samples of tonsil were teased to obtain a suspension of cells which was depleted of red cells to provide a leucocyte-enriched preparation, as described for blood leucocytes.

\section{RESULTS AND DISCUSSION}

While the primary objective of this study (to generate fibroblast specific mAbs) was not fully achieved, we have generated and performed an initial characterisation of a panel of 34 stromal specific mAbs. Tab. 1 summarises the

A
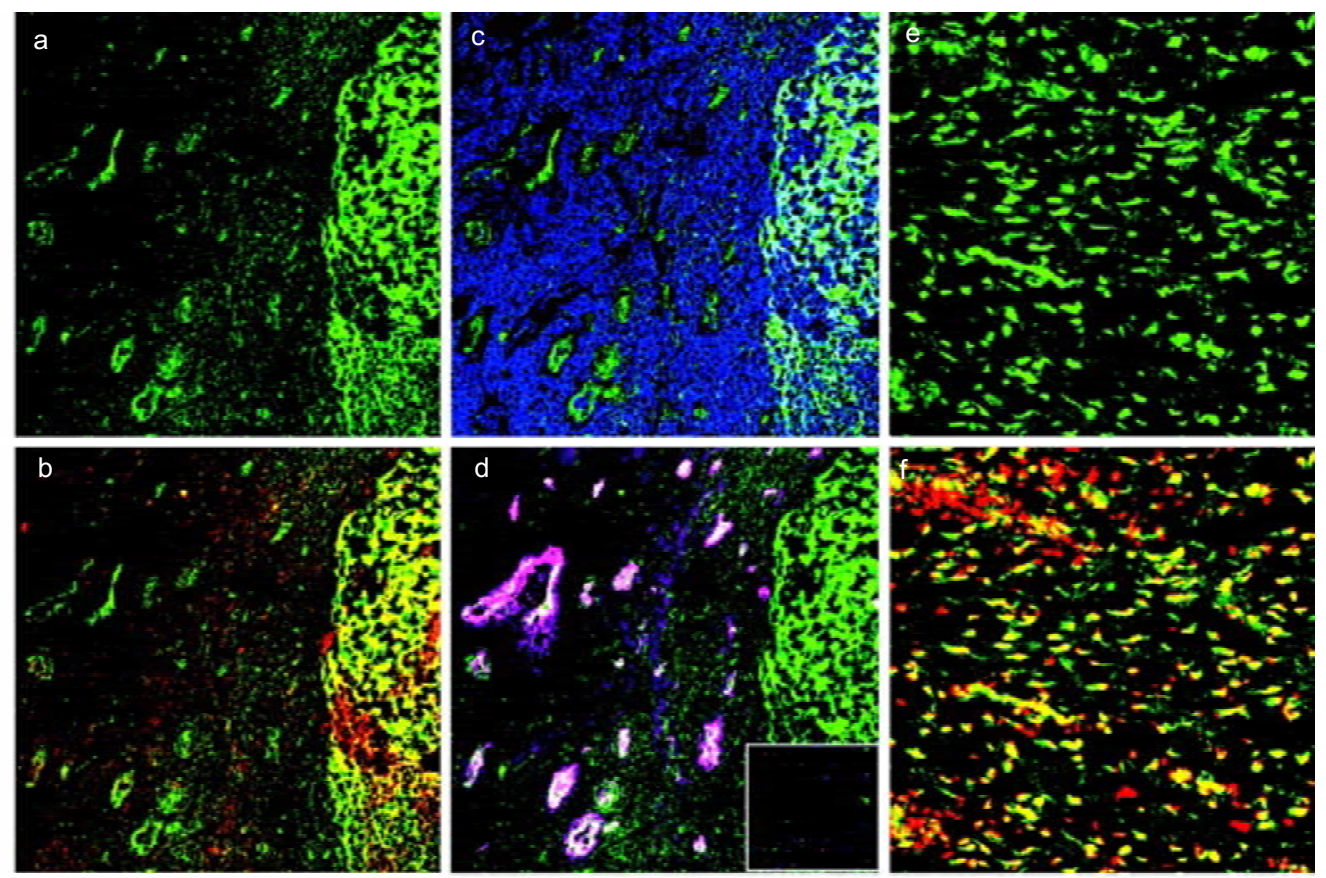

B

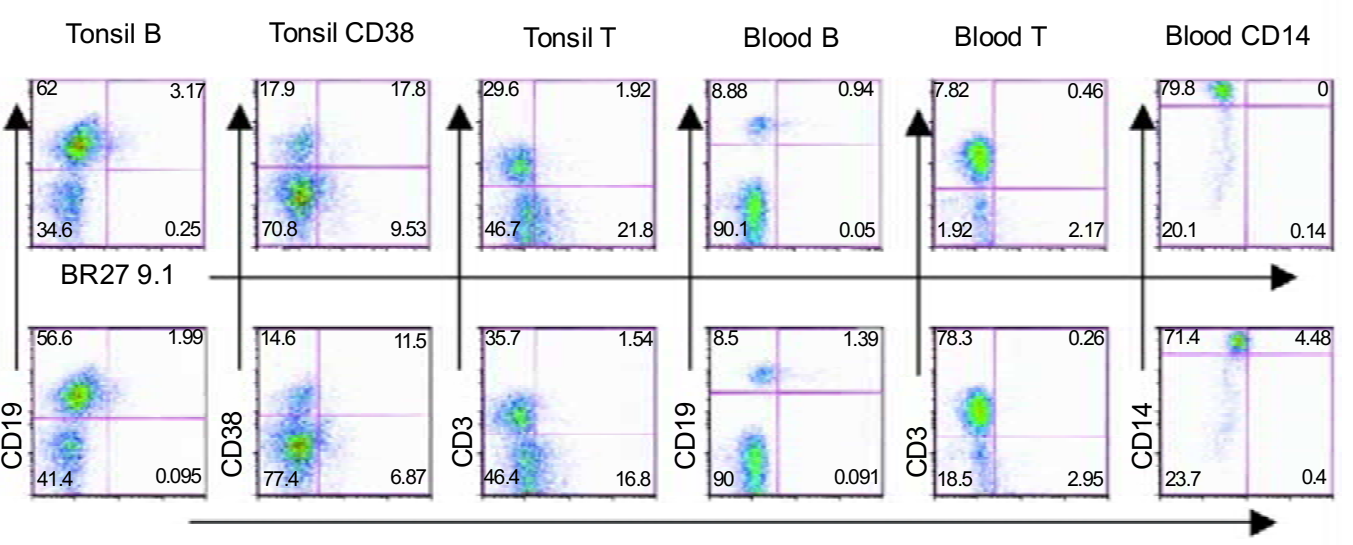

Lontrol

Fig. 2 More restricted stromal expression by mAb BR27 9.1. (A) BR27 9.1 (green) staining in tonsil (a-d) and rheumatoid synovium $(\mathrm{e}, \mathrm{f})$ in comparison with (b) 8D6 (red) (CD320); (c) CD45; (blue) (d) vWF (pink) and (f) nuclei (red). Co-incident staining appears as yellow (b and f), cyan (c) or white (d). BR27 9.1 has extensive staining of follicular dendritic cells (8D6 ${ }^{+}$) that are also CD45 $5^{+}$other $\mathrm{CD}^{+} 5^{+}$cells are not BR27 9.1 . BR27 9.1 also stains $v \mathrm{VF}^{+}$vessels but not epithelium (data not shown). Inset in (d) is negative control of secondary antibodies. Magnification: $\times 25$. (B) Lower panels: FACS data showing no surface expression of this antibody on forward and side scatter gated leucocytes that are CD19-APC ${ }^{+}, \mathrm{CD} 38-\mathrm{PE}^{+}, \mathrm{CD} 3-\mathrm{CyChrome}{ }^{+}$or CD14-PE ${ }^{+}$. Lower row is the secondary only negative control. 
number of clones obtained from each fusion and the number of clones selected for further investigation. The low immunogenicity of the fibroblasts used for immunisation is indicated in that the fusions themselves were successful in terms of the number of clones produced but overall only $\sim 7.9 \%$ of colonies had reactivity with rheumatoid fibroblasts as demonstrated by the immunohistochemical screen on synovial tissue. The inherently low immunogenic nature of these cells is not surprising and is an important consideration given the role of these cells in providing the backbone of the microenvironment wherein immune responses occurs. Future immunisation protocols could compare various fibroblast-activation factors and may also use fibroblasts from different tissues to try and improve the yield of fibroblast-reactive clones.

The antibodies fell into the following categories with: i) a broad stromal expression pattern including fibroblast, endothelial, epithelial and follicular dendritic cells; ii) a slightly more restricted pattern being negative with epithelium; and iii) a more restricted pattern reacting with antigen expressed on fibroblasts from rheumatoid synovium, stromal cells within the extrafollicular areas of sections of tonsil and endothelial-associated cells. There was no overall difference in the pattern of broad or restricted mAbs generated between fusions regardless of whether fresh or frozen immunogen was used and also between patients.

Fig. 1 shows mAb BR 274.11 that detects an antigen found on many stromal cells, including epithelium, endothelium ( $\mathrm{VWF}^{+}$vessels), follicular dendritic cells $\left(\mathrm{CD} 320^{+}\right)$and fibroblasts, but not on $\mathrm{CD} 45^{+}$leucocytes. The FACS data confirms that there is no expression of this antibody on leucocytes from tonsil or blood. Fig. 2 gives an example of the more restricted stromal staining pattern given by BR-27 9.1 that does not have reactivity with epithelium.

Many mAbs were generated that exhibited endotheliumassociated staining and these were further characterised to determine if the vessels detected by these mAbs were lymphatic or vascular using relevant markers. LYVE-1, a receptor for extracellular matrix hyaluronan was used as a marker for lymphatic endothelium as it is preferentially expressed on lymphatic endothelium and on a proportion of blood vessels in the lung [4]. The marker used for vascular endothelium was von Willebrand factor (vWF), which is necessary for platelet adhesion, plug formation and binding to factor VIII. Fig. 3 shows an example of mAb BR28 30.10 that binds preferentially to an antigen found on vascular but not lymphatic endothelium. This mAb also has reactivity with stromal cells within tonsil (Fig. 3) and fibroblasts in rheumatoid synovium (data not shown).

To conclude an absolute fibroblast-specific antibody has

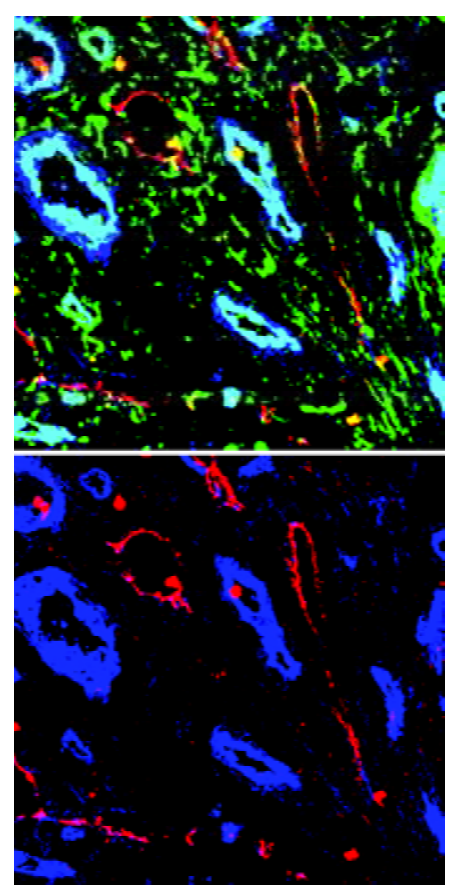

Fig. 3 A fibroblast-reactive $\mathrm{mAb}$ that also identifies vascular vessels. Tonsil sections showing (A) BR28 30.10 (green) binds preferentially to an antigen found on vascular $\left(\mathrm{vWF}^{+}\right)$endothelium (blue), coincident staining is shown as cyan. There is negligible coincident staining with lymphatic (red) LYVE-1 ${ }^{+}$vessels. BR28 30.10 also stains stromal cells (green only). (B) vWF (blue) and LYVE-1 (red) staining is compared in the absence of $\mathrm{mAb}$ staining. Magnification: $\times 25$

not been generated from our fusions to date. Of the many mAbs that identify fibroblasts and cells that are in close association with endothelial vessels, some may have reactivity with stromal pericytes [5]. Given the paucity of available fibroblast markers some interesting stromal-reactive antibodies have been generated that will help to initiate the phenotyping of fibroblasts and stromal cells in a similar way in which haematopoietic cells have been characterised. BR27 4.11 and BR27 9.1 show differential staining of epithelial cells, whilst both stain endothelium and FDC. BR28 30.10 identifies vascular endothelium and stromal cells other than FDC and epithelium. Further characterisation of the antibodies comparing different normal and inflamed tissues samples is ongoing work in our laboratories. The search for a fibroblast specific $\mathrm{mAb}$ that may provide a clue as to the role of these cells in chronic persistent rheumatoid arthritis is continuing.

\section{ACKNOWLEDGEMENTS}

We thank Drs Andy FILER and Nicole AMFT for rheumatoid synovium tissue samples. This work was supported by Celltech R\&D Ltd grant (No. RWJK08228). . 
Received, Aug 15, 2005

Revised, Sep 1, 2005

Accepted, Sep 15, 2005

\section{REFERENCES}

1 Kohler G, Milstein C. Continuous cultures of fused cells secreting antibody of predefined specificity. Nature 1975; 256: 495-7.

2 Chang HY, Chi JT, Dudoit S, et al. Diversity, topographic differentiation, and positional memory in human fibroblasts. Proc Natl Acad Sci U S A 2002; 99:12877-82.
3 Parsonage G, Falciani F, Burman A, et al. Global gene expression profiles in fibroblasts from synovial, skin and lymphoid tissue reveals distinct cytokine and chemokine expression patterns. Thromb Haemost 2003; 90:688-97.

4 Mouta Carreira C, Nasser SM, di Tomaso E, et al. LYVE-1 is not restricted to the lymph vessels: expression in normal liver blood sinusoids and down-regulation in human liver cancer and cirrhosis. Cancer Res 2001; 61:8079-84.

5 MacFadyen JR, Howarth OJ, Robertson D, et.al. Endosialin (TEM1) is a marker of stromal fibroblasts and is not selectively expressed on tumour endothelium. FEBS Lett 2005; 579:2569579:2569-75.

Edited by Heddy ZOLA (Guest editor) 